Europhysics Letters

PREPRINT

\title{
Suppressed absolute negative conductance and generation of high-frequency radiation in semiconductor superlattices
}

\author{
K. N. Alekseev ${ }^{1}$, M. V. Gorkunov ${ }^{1,2}$, N. V. Demarina ${ }^{3}$, T. Hyart $^{1}$, N. V. \\ AleXeEva $^{1}$ and A. V. Shorokhov ${ }^{1}$ \\ 1 Department of Physical Sciences, P.O. Box 3000, University of Oulu 90014, Finland \\ 2 Institute of Crystallography, Russian Academy of Sciences, Moscow 119333, Russia \\ 3 Nizhny Novgorod State University, 603950 Nizhny Novgorod, Russia
}

\begin{abstract}
PACS. 73.21.Cd - Superlattices.
PACS. 72.20.Ht - High-field and nonlinear effects.

PACS. 07.57.Hm - Infrared, submillimeter wave, microwave, and radiowave sources.
\end{abstract}

\begin{abstract}
We show that space-charge instabilities (electric field domains) in semiconductor superlattices are the attribute of absolute negative conductance induced by small constant and large alternating electric fields. We propose the efficient method for suppression of this destructive phenomenon in order to obtain a generation at microwave and $\mathrm{THz}$ frequencies in devices operating at room temperature. We theoretically proved that an unbiased superlattice with a moderate doping subjected to a microwave pump field provides a strong gain at third, fifth, seventh, etc. harmonics of the pump frequency in the conditions of suppressed domains.
\end{abstract}

Introduction. - There exists a strong demand for miniature, solid-state, room temperature operating sources and detectors of $\mathrm{THz}$ radiation $(0.3-10 \mathrm{THz})$. The need is caused by a rapid progress of $\mathrm{THz}$ sciences and technologies ranging from the astronomy to the biosecurity [1. Semiconductor superlattices (SLs) [2], operating in the miniband transport regime, are interesting electronic devices demonstrating properties of both nonlinear and active media. Nonlinearity of voltage-current (UI) characteristic of SL gives rise to a generation of harmonics of microwave and $\mathrm{THz}$ radiation [3, 4]. On the other hand, Bloch oscillations of electrons within a miniband of SL cause an appearance of negative differential conductance (NDC) for dc fields (voltages) larger than the critical Esaki-Tsu $E_{c}$ [2]. Remarkably, the static NDC should be accompanied by a small-signal gain for ac fields of very broad frequency range from zero up to several $\mathrm{THz}[5]$. The feasible device, employing such active media properties of dc-biased SL at room temperature, is known as $\mathrm{THz}$ Bloch oscillator. However, an existence of static NDC causes space-charge instabilities and a formation of high-field domains inside SL [6]. These domains are believed to be destructive for the Bloch gain. Recent top class experiment demonstrates only decrease in $\mathrm{THz}$ absorption, but still not gain, in an array of short dc-biased SLs [7. Therefore, it is important to consider modifications of this canonic scheme of generation in order to suppress electric domains but preserve the high-frequency

(C) EDP Sciences 
gain in SLs. Recently, the experimental realizations of SL oscillators with ac bias have been reported 8, 9].

In the present Letter, we analyze theoretically the scheme of high-frequency superlattice oscillator where instead of dc bias a quasistatic ac electric field is used. Because for typical SLs the characteristic scattering time at room temperature is of the order of $100 \mathrm{fs}$, the quasistatic interaction of ac field with miniband electrons is well defined for the microwave frequencies. SL is placed into the resonator tuned to the frequency of generation. Here the ac field plays a twofold role: It suppresses the space-charge instability inside SL and simultaneously pumps an energy for generation and amplification at higher frequency.

The main idea of our scheme for suppression of space-charge instability is very simple. Suppose that the frequency of the pump field $\omega$ is larger than the inverse characteristic time of domain formation $\tau_{d o m}^{-1}$. This requirement, $\omega \tau_{d o m} \gg 1$, imposes the lower limit for the values of pump frequency $\omega$ in our scheme, which, however, still easily belongs to the microwave range. Then, we show that the electric instability in the ac-driven superlattice arises if the slope of dependence of the time-averaged current on the applied dc bias is negative (NDC for the time-average current). We note that the only possibility to have NDC for the time-average current in the limit of small dc bias is related to the existence of absolute negative conductance (ANC), when the direction of dc current is opposite to its direction in a normal conductor (fig. 11). ANC is known in various systems [10, but this nonequilibrium phenomenon requires very special conditions, which as rule are quite difficult to satisfy. In particular, only a few combinations of quasistatic ac fields can induce ANC in SL. Thus, a high electric stability of the oscillator's scheme is based on a simplicity of ANC state suppression in unbiased or weakly dc-biased SLs.

We prove that within this stabilization scheme the only way to get gain for both small- and large-signals, is to tune a resonator to some particular harmonics of $\omega$ (fig. 22). The physical processes in SL contributing the the net gain are the parametric amplification of the field in resonator, seeded by the the frequency multiplication of the pump field, as well as the nonparametric absorption controlling the suppression of domains.

Suppression of domains. - We consider a response of electrons, belonging to a single miniband of SL, to the action of strong pump field $E_{p}=E_{d c}+E_{\omega} \cos \omega t$, where dc bias $E_{d c}$ is small and ac field is quasistatic $\omega \tau<1$ ( $\tau$ is the intraminiband relaxation time). This pump field creates the voltage $U_{p}=E_{p} L$ across SL of the total length $L$. We suppose also that due to a nonlinear character of miniband transport, a signal with amplitude $E_{1}<E_{\omega}$ and frequency $\omega_{1}>\omega$ is generated. The total voltage across SL $U(t)$ is

$$
U=U_{p}(t)+U_{s}(t)=\left(U_{d c}+U_{\omega} \cos \omega t\right)+U_{1} \cos \omega_{1} t
$$

For $\omega \tau<1$, the dependence of the current through SL $I(t)$ on the ac voltage $U(t)$ can be well described by the Esaki-Tsu formula

$$
I=2 I_{p}\left(U / U_{c}\right) /\left[1+\left(U / U_{c}\right)^{2}\right]
$$

where $U_{c}=(\hbar L) / e a \tau$ is the critical voltage ( $a$ is the SL period), and $I_{p} \equiv I\left(U_{c}\right)$ is the peak current [2]. Now we turn to the derivation of electric stability conditions of SL device. For $\omega \tau<1$ a space-time evolution of the electron density $\rho(x, t)$, the current density $j(x, t)$ and the field $E(x, t)$ inside SL, driven by the given voltage $U(t)$, can be well described by the drift-diffusion model [11]. Set of equations consists of the current equation $j=e \rho V(E)-$ $D(E) \partial \rho / \partial x$, the Poisson equation $\partial E / \partial x=4 \pi \epsilon^{-1}(\rho-N)$ with $N$ being the doping density, the relation to the applied voltage $U(t)=\int_{0}^{L} E(x, t) d x$, and the continuity equation $e \partial \rho / \partial t+$ 


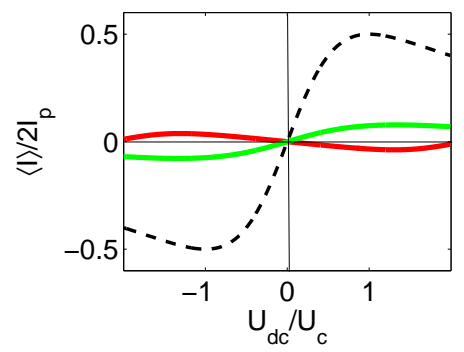

Fig. $1-$ [color online] Dependence of time-averaged current $\langle I\rangle$ on dc voltage $U_{d c}$ for the pump $U_{\omega} / U_{c}=9$ and for different strengths of signal $U_{1}$ at frequency $7 \omega$ : Positive slope for $U_{1} / U_{c}=0.9$ (green online), negative slope and $\mathrm{ANC}$ for $U_{1} / U_{c}=6.3$ (red online). Esaki-Tsu characteristic $\left(U_{\omega}=0\right)$ is shown as dashed line.

$\partial j / \partial x=0$ [12. Dependence of the electron velocity $V$ on the local field $E(x, t)$ is determined by the Esaki-Tsu formula as $V(E)=2 V_{p}\left(E / E_{c}\right) /\left[1+\left(E / E_{c}\right)^{2}\right]$ with $E_{c}=\hbar / e a \tau$ and $V_{p} \equiv$ $V\left(E_{c}\right)$. Following the Einstein relation the diffusion coefficient is $D=k_{B} T V(E) / E$. If the amplitude of $U(t)>U_{c}$, space-charge instabilities periodically arise during the part of period of ac voltage $T=2 \pi / \omega$ when the SL is switched to the state with NDC.

We suppose that condition $\omega \tau_{\text {dom }} \gg 1$ is satisfied. This inequality guarantees that a charge accumulated during $T$ is always limited. In this situation, fluctuations of $j$ and $\rho$ are small and we can use linear analysis investigating stability of the system. The characteristic time $\tau_{d o m}$ is of the order of the dielectric relaxation time $\sim\left(\omega_{p l}^{2} \tau\right)^{-1}\left(\omega_{p l}=\left(4 \pi e^{2} N / \epsilon m_{0}\right)^{1 / 2}\right.$ is the miniband plasma frequency, $N$ is the doping density, $m_{0}$ is the electron mass at the bottom of miniband, $\epsilon$ is the averaged dielectric constant of SL) [12. In more accurate approach $\tau_{\text {dom }}$ depends on the amplitude of ac field. Our numerical simulations demonstrate that for typical semiconductor SL with $N \simeq 10^{16} \mathrm{~cm}^{-3}$ accumulated charge is small $\left(\omega \tau_{\text {dom }} \gtrsim 1\right)$ if $\omega / 2 \pi \gtrsim 100 \mathrm{GHz}$. Decreasing doping density $N$ one can satisfy the condition using lower pump frequencies.

Linearizing the drift-diffusion model equations, we find that small fluctuations of spacecharge with a long wavelength will not grow, if the dependence of the time-averaged current $\langle I\rangle$ on the dc voltage $U_{d c}$ has positive slope, i.e.

$$
\Lambda=\left\langle I^{\prime}[U(t)]\right\rangle>0,
$$

where averaging $\langle\ldots\rangle$ is performed over period $T$, prime means first taking the derivative in respect to $U_{d c}$ and then assuming $U_{d c} \rightarrow 0$. Here and in what follows, we will consider the current $I$ and the voltage amplitudes $U_{\omega}$ in units of $I_{p}$ and $U_{c}$, correspondingly. Combining eqs. (3) and (2) for the quasistatic pump $U=U_{p}(t)$, we get $\Lambda_{p} \equiv \Lambda\left(U_{p}\right)=\left(1+U_{\omega}^{2}\right)^{-3 / 2}$, which is always positive for all $U_{\omega}$. Therefore, SL driven by a monochromatic and quasistatic ac field is stable against small fluctuations of charge or field.

We also considered the influence of signal fields on the stability of the system calculating numerically the increment $\Lambda$ for $U(t)$ given by eq. (1) with $\omega_{1}=n \omega t$. For $n=3$ we found that $\Lambda>0$ for all $U_{1} \leq 10$. Nevertheless, the UI characteristics with ANC, and as a consequence with $\Lambda<0$, can exist for some particular values of $U_{\omega}$ and $U_{1}$ in the cases $n=5$ and $n=7$ (fig. (1). However, for the practically important range $U_{1}<U_{1}^{s t}$ (see next section), we still found no ANC.

We should distinguish our scheme of electric stabilization based on avoidance of ANC in unbiased SLs from the free of domains Limited Space Accumulation (LSA) regime, which is 


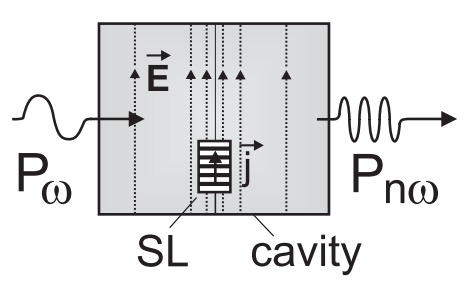

Fig. 2 - Schematic representation of the domainless oscillator: Generation at $n=3,7 \ldots$.

well-known in the Gunn diodes (see [13] for the theory and 14] for the theory and experiment) and can be applied to SLs as well [15]. This LSA regime works only for a large dc bias and for a large ac field in the resonator. In contrast, within our scheme it is possible to avoid a formation of domains even for small-amplitude fields in resonator.

Small-signal gain. - In the oscillator, SL is placed in a resonator providing a feedback only for the signal with frequency $\omega_{1}$. Absorption of the signal field in SL is defined as $A=\left\langle I[U(t)] \cos \left(\omega_{1} t\right)\right\rangle_{t}$, where $U(t)$ is given by (11) and averaging $\langle\ldots\rangle_{t}$ is performed over a period that is common for both $U_{p}(t)$ and $U_{s}(t)$. Gain corresponds to $A<0$.

In our case the pump is always quasistatic $(\omega \tau<1)$, but an interaction of the signal field with miniband electrons can be both quasistatic for sub- $\mathrm{THz}$ frequencies or dynamic for $\omega_{1} \tau \gtrsim 1$. Accordingly, to find $I(U)$ for $\omega \tau<1$ and $\omega_{1} \tau<1$ we can use the Esaki-Tsu eq. (2), but have to use the formal exact solution of Boltzmann transport equation [16] if $\omega_{1} \tau \gtrsim 1$. Surprisingly, in our situation both these approaches give quite similar physical results, therefore we focus mainly on more simple quasistatic calculations and only briefly discuss a gain for signal with $\omega_{1} \tau \gtrsim 1$.

For a small signal $U_{1} \ll U_{\omega}$, we expand $I(U) \approx I\left(U_{p}\right)+I^{\prime}\left(U_{p}\right) \times U_{s}$ and substitute this expression in the definition of absorption $A$. We see that because of averaging over time the absorption $A$ is strongly dependent on the ratio of frequencies $\omega_{1}$ and $\omega$. First, if $\omega$ and $\omega_{1}$ are incommensurable, we have $A=A_{\text {inc }} \equiv\left\langle I^{\prime}\left(U_{p}\right)\right\rangle U_{1} / 2$. Obviously, for the Esaki-Tsu dependence $A_{\text {inc }}=\Lambda_{p} U_{1} / 2>0$. Moreover, $A>0$ also for the probe with $\omega_{1} \tau \gtrsim 1$. Second, if $\omega_{1}=n \omega / 2$ ( $n$ is an odd number), we found that always $A>0$ for $\Lambda_{p}>0$. Thus, no gain at incommensurate or half-integer frequencies is possible in the conditions of suppressed domains.

Next, we consider the case when the probe frequency is $\omega_{1}=n \omega$ (fig. 21). In this case, the total absorption $A$ is the sum of three terms: Defined above $A_{\text {inc }}$, as well as $A_{h} \equiv$ $\left\langle I\left(U_{p}\right) \cos (n \omega t)\right\rangle$ and $A_{c o h} \equiv\left\langle I^{\prime}\left(U_{p}\right) \cos (2 n \omega t)\right\rangle U_{1} / 2$, which can be called the incoherent, the harmonic and the coherent absorption components, correspondingly [17.

We see that while $A_{c o h}$ and $A_{i n c}$ are dependent on both $U_{\omega}$ and $U_{1}$, the term $A_{h}$ is a function of only pump strength $U_{\omega}$, and therefore $A_{h}$ gives the main contribution to the absorption of a weak probe. For the Esaki-Tsu dependence we find $A_{h}=(-1)^{k}\left[2(b-1)^{2 k+1}\right] / b U_{\omega}^{2 k+1}$, where $b=\left(1+U_{\omega}^{2}\right)^{1 / 2}$ and $2 k+1=n(k=1,2,3 \ldots)$. This equation describes just odd harmonics of the current (cf. Eq. 17 in [4); in the limit of weak pump $U_{\omega} \ll 1$ it takes familiar form $\propto U_{\omega}^{n}$ 3. Importantly, $A_{h}$ is negative for the odd values of $k$ (fig. [3 ). Therefore, generated harmonics with $n=3,7, \ldots$ can provide seeding gain for an amplification of a probe field. In quasistatic picture, it has no threshold in the amplitude of pump $U_{\omega}$. Thus, if the pump amplitude is less than the critical Esaki-Tsu voltage, the gain at $3 \omega$ will be not accompanied by the space-charge instabilities in SL.

Nonparametric effects in absorption are described by the term $A_{i n c} \propto \Lambda_{p}$. It is always 

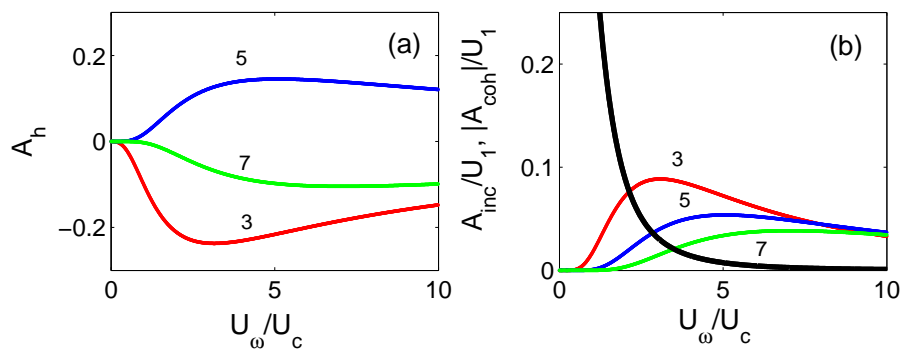

Fig. 3 - [Color online] (a) Amplitudes of the 3d (red online), 5th (blue online) and 7th (green online) harmonics of the current, $A_{h}$, as functions of the pump amplitude $U_{\omega}$. (b) Incoherent $A_{\text {inc }}$ (black) and absolute values of coherent $-A_{c o h}$ components of small-signal absorption at $3 \omega, 5 \omega$ and $7 \omega$, as functions of pump $U_{\omega}$.

positive because of space-charge stabilization. The term $A_{c o h}$ describes a parametric amplification of the probe field due to a coherent interaction of the pump and the probe fields in SL. Numerical calculation of the integral demonstrates that $A_{c o h}$ is always negative for odd $n=3,5,7$; its absolute value is larger than $A_{\text {inc }}$ for a large enough pump strengths (fig. [3b). In the limit of weak pump $U_{\omega} \ll 1$, we find $A_{c o h} \propto U_{\omega}^{2 n} U_{1}$. Now we can describe the amplification of a weak signal at $3 \omega$ under the action of a weak pump. Third harmonic of a weak pump is generated at the cubic nonlinearity of SL characteristic, then it can get a seeding gain at the same nonlinearity $\left(A_{h} \propto U_{\omega}^{3}\right)$, but the next step of parametric gain, $A_{c o h}$, uses already the seventh order $\left(\simeq U^{7}\right)$ nonlinearity in the $I(U)$ dependence.

We should underline that the sign of generated current at the frequency of harmonic plays a very important role in the process of amplification. Really, let us compare the action of SL oscillator tuned either to $3 \omega$ or to $5 \omega$ modes (fig. 2). Net gain at $3 \omega$ is quite large, what can provide an effective operation of the oscillator. On the other hand, a radiation at 5 th harmonic also can be generated in SL. However, in contrast to the case of the $3 \mathrm{rd}$ harmonic, it cannot be further amplified (because $A_{h}>0$ and $A_{h}>\left|A_{\text {coh }}\right|$ ) and field in the cavity, tuned to $5 \omega$, eventually evolves to zero.

Large-signal gain. - We calculate the large-signal absorption $A\left(U_{\omega}, U_{1}\right)$ using two methods: (i) simple 1D calculations with Esaki-Tsu characteristic (eqs. (2) and (1D) and (ii) 3D single-particle Monte Carlo computations with an account of electron scattering at optical and acoustic phonons [18]. For the Monte Carlo computations we consider GaAs/AlAs SL of the period $a=6.22 \mathrm{~nm}$ and the miniband width $\Delta=24.4 \mathrm{meV}$. Static UI characteristic of this SL can be well described by the Esaki-Tsu formula with $E_{c}=4.8 \mathrm{kV} / \mathrm{cm}$ and $\tau=220 \mathrm{fs}$. We took $\omega / 2 \pi=100 \mathrm{GHz}$. These are our default parameters.

The dependence $A\left(U_{\omega}\right)$ for $3 \omega$-generation and for different values of the relative probe amplitude, $\eta=U_{1} / U_{\omega}$, are shown in fig. [. Results of simple 1D theory and 3D Monte Carlo simulations are in a good agreement. For $\eta=0$ and for small $\eta \ll 1$, the dependence of $A\left(U_{\omega}\right)$ follows to the corresponding dependencies for the seeding gain, $A_{h}\left(U_{\omega}\right)$, and the small-signal gain. With a further increase of $\eta$, the gain decreases and finally the absorption becomes zero for some $\eta=\eta_{0}: A\left(U_{\omega}, \eta_{0}\right)=0$. The value $\eta_{0}^{2}=\left[U_{1}^{s t} / U_{\omega}\right]^{2}$ determines the maximal device efficiency for the given pump (here $U_{1}^{s t}$ is the voltage amplitude corresponding to the

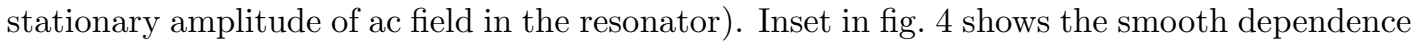
of $\eta_{0}$ on the pump amplitude. Generation with low efficiency (less than 5\%) is possible even without static NDC, for $U_{\omega}<1$. The efficiency reaches its maximal value of $23 \%$ at $U_{\omega} \simeq 4$. 


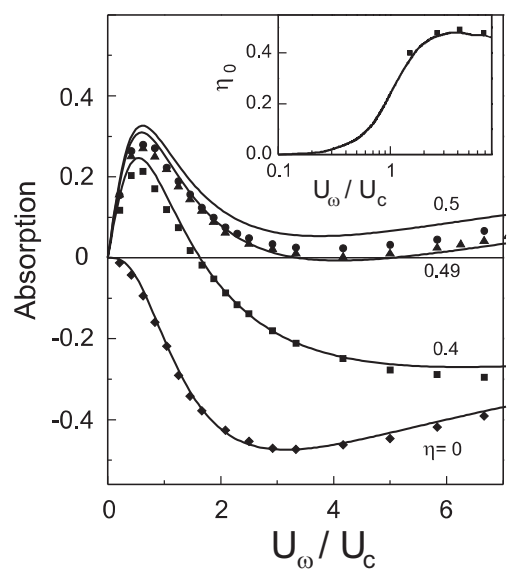

Fig. 4

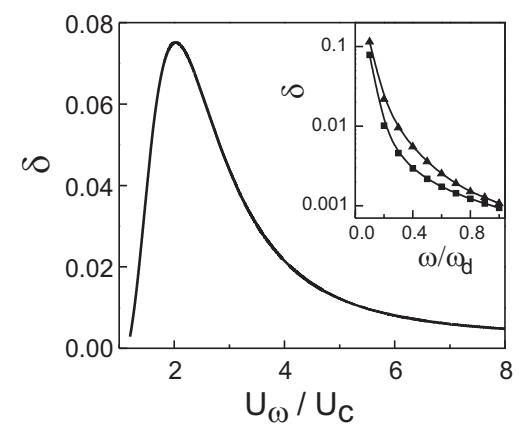

Fig. 5

Fig. 4 - The dependence of absorption at $3 \omega$ on the pump amplitude $U_{\omega}$ for different relative probes, $\eta$. Inset: Maximal relative amplitude of the signal allowing gain, $\eta_{0}$, as a function of the pump. Calculation with Esaki-Tsu characteristic (solid) and Monte Carlo technique (symbols).

Fig. 5 - Regime of suppressed domains in SL oscillator. Relative reduction of gain at 3d harmonic $\delta$ vs voltage amplitude $U_{\omega}$ for $\omega / \omega_{d}=0.1$ and $\eta=U_{1} / U_{\omega}=0.1$. Inset: $\delta$ as a function of the ratio $\omega / \omega_{d}$ for $U_{\omega} / U_{c}=2, \eta=0$ (squares) and $\eta=0.4$ (triangles).

For $7 \omega$-oscillations the maximum of $\eta_{0}^{2}$ is $4 \%$.

Numerical evidence. - We directly calculate the influence of inhomogeneous distributions of space-charge on the gain in SL. We numerically solve the drift-diffusion model for the time-dependent voltage $U(t)$ (eq. (1) ) with $U_{d c}=0$ and determine the absorption at the $n$th harmonic as $A_{d}=\left\langle\left\langle\left(j(x, t) / j_{0}\right) \cos n \omega t\right\rangle\right\rangle$, where $j_{0}=e V_{p} N$ and averaging $\langle\langle\ldots\rangle\rangle$ is performed both over the period $T$ and the length $L$. In the computations a spatial fluctuation of charge distribution was caused by a local Gaussian-profiled deviation of $E_{c}$ (or $\tau$ ). We observed a periodic spatial accumulation of charges during a part of period $T$. We computed the relative decrease in gain, $\delta=\left(A-A_{d}\right) / A$, for different harmonics $n$ and pumps $U_{\omega}$. Dependence of $\delta$ on $U_{\omega}$, for SL of 130 periods and for $\omega / \omega_{d}=0.1\left(\omega_{d} \equiv \omega_{p l}^{2} \tau\right)$, is shown in fig. [5 In fig. 5 the reduction of gain due to charge accumulation is $2 \%$ for optimal $U_{\omega}=4$ and it is less than $8 \%$ overall. For our default parameters, the value $\omega / \omega_{d}=0.1$ corresponds to the doping $N=2 \times 10^{16} \mathrm{~cm}^{-3}$. In this situation $\omega \tau_{d o m} \simeq 1$. We found that with an increase of $\omega / \omega_{d} \propto \omega \tau_{d o m}$, the value of $\delta$ quickly decreases (see Inset in Fig. 5). Therefore, for higher frequencies or lower doping $\left(\omega_{d} \propto N\right)$ providing $\omega / \omega_{d}>0.1$, the influence of charge accumulation on the gain is practically negligible.

Additionally, we also have performed the ensemble Monte Carlo simulations [19] to modell the oscillator with account of space-charge dynamics in SL. In general, these 3D simulations do confirm our main conclusions on the suppression of domains made in the framework of 1D drift-diffusion model.

Conclusion. - In summary, we derived the necessary and sufficient conditions for the suppression of space-charge instabilities in the high-frequency SL oscillators with a microwave pump. Our findings can provide simple tests to distinguish domainless and domain-mediated regimes of generation in these oscillators, such as a measurement of the dependence of the 
time-average current on the dc voltage or a comparison of operation at $3 \omega$ and $5 \omega$.

The domainless oscillator can operate at $\mathrm{THz}$ frequencies. Probably the most easily realizable case is to use a pump with $\omega$ near $100 \mathrm{GHz}$ to obtain an output in the important frequency band of hundreds of $\mathrm{GHz}$. In order to suppress electrical domains and to use resonator with a reasonable $Q$ (e.g., $Q \lesssim 100$ for generation at $3 \omega$ ), the doping of SL should be $\simeq 10^{16} \mathrm{~cm}^{-3}$.

In the recent experiments 8,9 , a generation at the third harmonic of a strong microwave pump of $100 \mathrm{GHz}$ have been reported in SL device. This generation has been first attributed [8] to the process of periodic formation of electric domains, but later it has been re-attributed to a parametric generation in [9]. In [8, 9] a heavy doped $\left(N=10^{18} \mathrm{~cm}^{-3}\right)$ SL was used, and therefore this device certainly operated in the regime with $\omega \tau_{d o m} \ll 1$, which is opposite to the condition of limited charge accumulation. In this situation, a sufficient charge accumulation during every cycle of pump field arises, and therefore SL can switch to ANC state, as has been shown for the case of microwave-driven Gunn diode in 20. We also saw such kind of ANC states in our simulations of SL oscillator within the drift-diffusion model. This ANC induced by the accumulated charge completely alters electric stability of SL device and results in an operational mode that is quite different from the mode based on an avoidance of ANC. Detailed theoretical analysis of the experiments [8, 9] goes beyond the scope of present paper.

$* * *$

This research was supported by Academy of Finland (grants 202697 and 109758), Emil Aaltonen Foundation, Programme of RFAE (grant 4629) and AQDJJ Programme of ESF.

Erratum. - Additional modelling of a high-frequency generation with consideration of a resonator tuned to $n \omega$ showed that the phase difference $\phi_{n}$ between the pump field $(\omega)$ and the signal field in resonator $(n \omega)$ can be either 0 for $n=3,7$ or $\pi$ for $n=5,9$ within the quasistatic approximation. Condition $A_{h}>0$ determines the phase shift $\phi_{5}=\pi$ between the pump field $(\omega)$ and the signal field in resonator $(5 \omega)$, but it does not prevent a growth of this mode, in contrast to our previous statement. Therefore, the amplitude of field $E_{1}$ in an ideal resonator tuned to $5 \omega$ in fact can grow until it reaches a stationary value $E_{1}^{s t}$ corresponding to zero value of the total absorption $A\left(E_{\omega}, E_{1}^{s t}\right)=0$, which is now defined as $A=\left\langle I[E(t)] \cos \left(n \omega t+\phi_{n}\right)\right\rangle_{t}$ for the total field $E(t)=E_{\omega} \cos (\omega t)+E_{1} \cos \left(n \omega t+\phi_{n}\right)$. The maximal efficiency $\eta_{0}^{2}=\left[E_{1}^{s t} / E_{\omega}\right]^{2}$ is $10 \%$. Next, following the necessary condition for suppression of domains [eq. (3)] the generation at $5 \omega$ is still possible in domainless mode.

\section{REFERENCES}

[1] Ferguson B. and Zhang X.-C., Nature Materials, 1 (2002) 26.

[2] Esaki L. and Tsu R., IBM J. Res. Dev., 14 (1970) 61.

[3] Esaki L. and Tsu R., Appl. Phys. Lett., 19 (1971) 246.

[4] Ignatov A. A. and Romanov Yu. A., Phys. Stat. Sol. B, 73 (1976) 327.

[5] Ktitorov S. A. et al., Sov. Phys. Solid State, 13 (1972) 1872.

[6] Ignatov A. A. and Shashkin V. I., Sov. Phys. JETP, 66 (1987) 526.

[7] Savvidis P. G. et al., Phys. Rev. Lett., 92 (2004) 196802.

[8] Klappenberger F. et al., Appl. Phys. Lett., 84 (2004) 3924.

[9] Renk K. F. et al., Phys. Rev. Lett., 95 (2005) 126801.

[10] Ros A. et al., Nature, 436 (2005) 928.

[11] Bonilla L. L. and Grahn H. T., Rep. Prog. Phys., 68 (2005) 577. 
[12] Volkov A. F. and Kogan S. M., Sov. Phys. Usp., 11 (1969) 881; Pamplin B. P., Contemp. Phys., 11 (1970) 1.

[13] Copeland J., Proc. IEEe, 54 (1966) 1479; J. Appl. Phys., 38 (1967) 3096.

[14] Altukhov I. V. et al., Sov. Phys. Semicond., 12 (1978) 172; Ibid., 13 (1979) 1356.

[15] Rieder B., Dissertation (University of Regensburg) 2004.

[16] Chambers R. G., Proc. Phys. Soc. (London) A, 65 (1952) 458; Budd H, Phys. Rev., 127 (1962) 4.

[17] For details of calculations, see Alekseev K. N. et al., cond-mat/0503216 preprint, 2005.

[18] For details of the method, see Demarina N. V. and RenK K. F., Phys. Rev. B, 71 (2005) 035341.

[19] Hockney R. W. and Eastwood J. W., Computer simulation using particles (McCraw-Hill, New York)1981.

[20] Banis T. YA. et al., Sov. Phys. Semicond., 5 (1972) 1727. 